\title{
Tremor severity in Parkinson's disease and cortical changes of areas controlling movement sequencing:
}

\section{A preliminary study}

(Running title: PD tremor severity and cortical changes)

\author{
Julián Benito-León, ${ }^{\mathrm{a}, \mathrm{b}, \mathrm{c}}$ J. Ignacio Serrano, ${ }^{\mathrm{d}}$ Elan D. Louis,,${ }^{\mathrm{e}}$ f, g Ales Holobar, ${ }^{\mathrm{h}}$

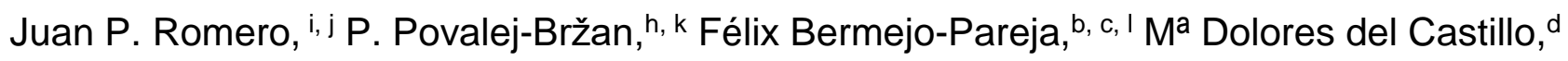 \\ Ignacio J. Posada, ${ }^{\text {a, c }}$ Eduardo Rocond
}

Department of Neurology, a University Hospital 12 de Octubre. Madrid, Spain; Center of Biomedical Network Research on neurodegenerative diseases (CIBERNED), b Spain; Department of Medicine, ${ }^{c}$ Faculty of Medicine, Complutense University, Madrid, Spain; Neural and Cognitive Engineering group, ${ }^{d}$ Centre for Automation and Robotics (CAR) CSICUPM, Arganda del Rey, Spain; Department of Neurology,e Yale School of Medicine; Department of Chronic Disease Epidemiology, ${ }^{f}$ Yale School of Public Health; Center for Neuroepidemiology and Clinical Neurological Research,g Yale School of Medicine and Yale School of Public Health, New Haven, CT, USA; Faculty of Electrical Engineering and Computer Science, ${ }^{h}$ University of Maribor, Maribor, Slovenia; Faculty of Biosanitary Sciences, ${ }^{i}$ Francisco de Vitoria University, Pozuelo de Alarcón, Madrid, Spain; Brain Damage Service, ${ }^{j}$ Hospital Beata Maria Ana, Madrid, Spain; Faculty of Health Sciences, ${ }^{k}$ Faculty of Health Sciences, University of Maribor, Maribor, Slovenia; Clinical Research Unit,' University Hospital 12 de Octubre, Madrid, Spain. 
*Corresponding author: Julián Benito-León, MD, PhD. Av. de la Constitución 73, portal 3, 7º Izquierda, 28821 Coslada, Madrid, Spain. E-mail: jbenitol67@gmail.com

Title character count: 98; Number of references: 63; Number of tables: 3; Number of figures: 4; Word count abstract: 203; Word count paper: 4,962. 
Abstract. There remains much to learn about the changes in cortical anatomy that are associated with tremor severity in Parkinson's disease (PD). For this reason, we used a combination of structural neuroimaging to measure cortical thickness and neurophysiological studies to analyze whether PD tremor was associated with cortex integrity. Magnetic resonance imaging and neurophysiological assessment were performed in 13 nondemented PD patients (9 women, 69.2\%) with a clearly tremor-dominant phenotype. Cortical reconstruction and volumetric segmentation was performed with the Freesurfer image analysis software. Assessment of tremor was performed by means of high-density surface electromyography (hdEMG) and inertial measurement units (IMUs). Individual motor unit discharge patterns were identified from surface hdEMG and tremor metrics quantifying motor unit synchronization from IMUs were defined. Increased motor unit synchronization (i.e., more severe tremor) was associated with cortical changes (i.e., atrophy) in dorsal premotor cortices, left posterior parietal cortex, left lateral orbitofrontal cortex, cingulate cortex bilaterally, left posterior and transverse temporal cortex, and left occipital lobe, as well as reduced left middle temporal volume. Given that the majority of these areas are involved in controlling movement sequencing, our results support Albert's classic hypothesis that PD tremor may be the result of an involuntary activation of a program of motor behavior used in the genesis of rapid voluntary alternating movements.

Keywords: Cortical thickness, Electromyography, Parkinson's disease, Magnetic resonance imaging, Tremor. 


\section{Significance Statement}

Little is known about possible changes in cortical anatomy in Parkinson's disease associated with the severity of tremor. For this reason, we used a combination of structural neuroimaging to measure cortical thickness and neurophysiological studies to analyze whether Parkinson's disease tremor was associated with cortex integrity. Increased motor unit synchronization correlated with cortical changes in areas controlling motor sequencing. Our results support Albert's classic hypothesis that Parkinson's disease tremor may be the result of an involuntary activation of a program of motor behavior used in the genesis of rapid voluntary alternating movements. 


\section{Introduction}

Parkinson's disease (PD) is a neurodegenerative disorder with a wide clinical spectrum, including several motor and non-motor features. Three-fourths of PD patients will experience tremor at some point during the disease (Zach et al. 2015). Although different types of tremor may occur in PD patients (Deuschl et al. 1998), the most common of these is rest tremor. This is a slow, biplanar, pill-rolling, 4-6 Hz tremor in the fully supported limb; the tremor decreases during movement (Chen et al. 2017; Deuschl et al. 1998). Despite the high prevalence of tremor among PD patients, it remains one of the most enigmatic hallmarks of this disease, which is an essentially a hypokinetic movement disorder. The pathophysiological mechanisms underlying tremor in PD remain incompletely understood despite extensive research. There are still insufficient data to answer a sizable number of basic questions about the pathogenesis of tremor in PD patients. For example, neither the action nor the rest tremor observed in PD correlate with the severity of other cardinal motor features, such as bradykinesia or rigidity, or with Hoehn and Yahr score or cognitive scores (Louis et al. 2001). Unlike other features of PD, such as bradykinesia, rigidity, postural abnormalities, gait difficulties, and other midline features, including changes in speech and facial expression, the severity of parkinsonian rest and action tremors are not related to the degree of dopaminergic denervation (Pirker 2003). Furthermore, patients with tremor dominant PD have slower progression of disability and a more favorable course than patients without tremor (Rajput et al. 2009). All these characteristics suggest a different pathophysiology underlying tremor dominant PD and non-tremor dominant PD. 
Basal ganglia dysfunction and striatal dopamine degeneration are most strongly related to bradykinesia and rigidity in $\mathrm{PD}$, and seem to be less associated with the genesis of tremor (Lewis et al. 2013). The circuit changes that mediate PD tremor, while likely differing from those underlying akinesia and rigidity, are not precisely known (Mure et al. 2011). Evidence suggests that PD tremor may be mediated by independent oscillating circuits primarily in the cerebello-thalamo-cortical pathways (Mure et al. 2011). Tremor may be generated by neural mechanisms that are actively working to compensate for akinesia and rigidity (Qasim et al. 2016).

Using PET, Parker et al.(Parker et al. 1992) studied seven PD patients both during the absence of tremor, e.g. during thalamic nucleus ventralis intermedius electrical stimulation, and in presence of tremor. They observed an activation of the sensory-motor cortex, as well as an involvement of the supplementary motor area and the cortico-cerebellar pathways in resting tremor (Parker et al. 1992). Another PET study reported cerebellar hypermetabolism associated with tremor (Deiber et al. 1993). A resting state PET with 18F-fluorodeoxyglucose study found that PD patients with tremor had an increased expression of a metabolic network comprising the thalamus, pons, and premotor cortical areas (Antonini et al. 1998). Another 18F-fluorodeoxyglucose PET study revealed a negative correlation between tremor and activity in the putamen and cerebellar vermis (Lozza et al. 2002). A magnetoencephalographic study demonstrated tremor-related oscillatory activity within a cerebral network, with abnormal coupling in a cerebello-diencephalic-cortical loop and cortical motor (primary motor cortex, cingulate/supplementary motor area, lateral premotor cortex) and sensory (secondary somatosensory cortex, posterior parietal cortex) areas contralateral to the tremor hand (Timmermann et al. 2003). Another 
magnetoencephalography study found that 3 to $6 \mathrm{~Hz}$ tremor in PD was accompanied by rhythmic electrical activation at the diencephalic level and in lateral premotor, somatomotor, and somatosensory cortex (Volkmann et al. 1996). Overall, these findings suggest that there is a common network of cerebral areas involved in PD tremor and voluntary repetitive movements (Duval et al. 2016), pointing towards the Albert's classic hypothesis that considered PD tremor as an involuntary activation of a motor behavior program for the voluntary production of rapid alternating movements (Alberts 1972). In general, motor behavior programs depend on the accurate control of movement sequencing.(Willingham 1998)

The identification of a specific pattern of cortical involvement associated with the severity of parkinsonian tremor would add to the available evidence concerning the role of the cortex in the genesis of tremor in PD. To our knowledge, the relationship between structural or corticometric changes and the severity of PD tremor has not been sufficiently explored and elucidated. There is only one previous study, involving mid-stage PD patients, that did not find any correlation between cortical thickness and the tremor subscore (on and off) of the motor section of the Unified Parkinson's Disease Rating Scale (UPDRS-m) (Deng et al. 2016). We hypothesized, however, that the anatomical integrity of the cerebral cortex is associated with tremorgenic activity of PD, especially in frontal and parietal areas involved in movement sequencing. Towards that purpose, we used a combination of structural neuroimaging to measure cortical thickness and neurophysiological studies (high-density electromyography [hdEMG] and inertial measurement units [IMUs]) to analyze whether the tremor of PD was associated with cortex integrity. 


\section{Methods}

\section{Ethical aspects}

All the participants included in the study gave their written informed consent after full explanation of the procedure. The study, which was conducted in accordance with the principles of the Helsinki declaration of 1975, was approved by the ethical standards committee on human experimentation at the University Hospital "12 de Octubre" (Madrid). Written (signed) informed consent was obtained from all enrollees.

\section{Participants}

PD patients were consecutively recruited from October 2012 to July 2013 from the outpatient neurology clinics of the University Hospital "12 de Octubre” in Madrid (Spain). Two neurologists with expertise in movement disorders (JB-L and JPR) examined the patients and used the UPDRS-m for those with PD (Martinez-Martin et al. 1994). Diagnoses of PD were assigned by the two neurologists (JB-L and JPR) using the UK PD Society Brain Bank Clinical Diagnostic criteria (Hughes et al. 1992). Clinical inclusion criteria were: (1) tremordominant PD phenotype; (2) Hoehn and Yahr stage of 1 or 2; and (3) absence of structural abnormalities in the brain, affecting gray or white matter, on conventional magnetic resonance imaging $(\mathrm{MRI})$. Patients with history of stroke, epilepsy, or head injury were excluded. Furthermore, based on a detailed cognitive test battery (Benito-Leon et al. 2015), we excluded patients with Diagnostic and Statistical Manual of Mental Disorders (DSM)-IV criteria for dementia (American Psychiatric 1994). Clinical characteristics were obtained from 
review of records from their outpatient neurological care. The PD patients were also followed at regular intervals $(3,6$, or 12 months, based on clinical need).

Healthy controls were recruited either from relatives or friends of the health professionals working at the University Hospital "12 de Octubre" of Madrid (Spain) or among the relatives of patients who came to the neurological clinics for reasons other than PD (e.g., headache, dizziness). None reported having a first-degree or second-degree relative with PD. Each control was examined by two neurologists (JB-L and JPR), who were blinded to the MRI results, to further rule out any neurological or other serious conditions, including movement disorders, dementia, stroke, epilepsy, or head injury.

All of the neurophysiological and MRI studies (see below) were performed during the same week and while taking their regular daily medication for PD.

\section{Neurophysiological procedures}

Recordings were carried out while patients were seated in a comfortable armchair, in a dimly illuminated room. Postural and rest tremor were simulated by asking the subject to perform the tasks described in Table 1. The patients were instructed to stay relaxed and keep their gaze fixed on a wall at $\sim 2-\mathrm{m}$ distance. Those with mild tremor severity were asked to mentally count backward during the recordings to enhance their tremor. The recordings lasted between $40 \mathrm{~s}$ and $4 \mathrm{~min}$. For the analysis, we selected the $30 \mathrm{~s}$ interval during which tremor had maximum amplitude. 


\section{Assessment of tremor in wrist movements}

Hand movements were measured by two pairs of IMUs (Technaid S.L., Madrid, Spain), placed on the dorsum of each hand and on the distal third of each forearm. The raw IMUs signals were sampled at $100 \mathrm{~Hz}$ by a 12 -bit $\mathrm{A} / \mathrm{D}$ converter, and low pass filtered $(<20$ $\mathrm{Hz}$ ). They were stored and processed off-line using MATLAB (The MathWorks, Natick, MA).

In the offline analysis, the difference between the IMUs signals from the hand and the corresponding forearm was calculated. Welch's power spectral density (PSD) was then assessed from 1 second long signal segments. The frequency with the maximum PSD on the interval form 0 to $12 \mathrm{~Hz}$ was selected as the basic tremor frequency and up to three tremor harmonics were identified as the maximums in PSD at the double, triple and quadruple tremor frequency, respectively, with tolerance of $\pm 0.5 \mathrm{~Hz}$. In order to emphasize the relative contributions of higher harmonics, the amplitudes of detected PSD peaks were expressed in logarithmic scale and then averaged, yielding the mean logarithmic power of tremor.

Finally, mean logarithmic tremor power was averaged over all the postural (arms outstretched [AO], arms supported + postural tremor elicited [PO]), arms outstretched with weights (WE), and rest (RE) tasks, see Table 1). In addition, the difference between the mean logarithmic tremor PSD averaged across all the postural tasks (AO, PO, WE) and the mean logarithmic tremor PSD averaged across the rest $(R E)$ tasks was calculated. The following tremor features were therefore extracted from the IMUs recordings ( $\mathrm{Br}$ et al. 2017):

- Mean logarithmic PSD of the tremor harmonics, averaged over the AO, PO, WE and RE tasks in the dominant and non-dominant tremor hand, respectively. This metric reflects 
the net effect of all the muscles that affect the wrist movement. Generally speaking, the higher the metric's value, the higher the synchronization of motor unit activity in muscles controling the wrist.

- Mean logarithmic PSD of the tremor harmonics, averaged over the AO, PO, and WE tasks with respect to RE task in the dominant tremor hand and in the non-dominant tremor hand, respectively. This metric quantifies how the synchronization of muscles and motor units changes when patients switch from rest to postural task. Positive values indicate stronger synchronization in postural task than in rest condition, whereas negative values indicate the stronger synchronization in rest than in postural tasks.

\section{Surface hdEMG signals}

Surface hdEMG signals were recorded from the wrist flexors and extensors with $5 \times 13$ electrode arrays (LISiN-OT Bioelettronica, Torino, Italy, $8 \mathrm{~mm}$ interelectrode distance). The electrode arrays were centred over flexor carpi radialis and extensor digitorum communis, respectively. Before electrode placement, the skin was cleaned and lightly abraded using abrasive paste (Meditec-Every, Parma, Italy). The electrode-skin contact was improved by filling each of the electrode cavities in the array with conductive gel (Meditec-Every, Parma, Italy). A soaked bracelet placed around one of the wrists was used as reference electrode. The bipolar surface hdEMG recordings were amplified, band-pass filtered ( $3 \mathrm{~dB}$ bandwidth, $10-750 \mathrm{~Hz}$ ) and sampled at $2048 \mathrm{~Hz}$ and 12-bit resolution (LISiN-OT Bioelettronica, Torino, Italy). 
The Convolution Kernel Compensation (CKC) algorithm was used to decompose the hdEMG signals into motor unit spike trains (Holobar et al. 2009). The Pulse-to-Noise Ratio (PNR) metric was used to assess the accuracy of each indivodual motor unit spike train estimation (Holobar et al. 2014). The motor units with $\mathrm{PNR}<30 \mathrm{~dB}$ (corresponding to accuracy of $<90 \%$ ) were discarded (Holobar et al. 2014).

Pair-wise motor unit synchronization has been quantified by assessing the distribution of backward and forward motor unit recurrence times in pairs of simultaneously active motor units (De Luca et al. 1993). The forward (backward) recurrence time was defined as the distance from the current motor unit discharge to the first next (closest previous) discharge of paired motor unit (Glaser et al. 2017). For each pair of identified motor units, we computed the $99 \%$ confidence limit in a histogram of motor unit recurrence times, assuming their uniform distribution in absence of pathological tremor (Glaser et al. 2017). We then defined the percentage of concurrent motor unit discharges as the ratio between the peak area exceding this limit and the sum of all the histogram bins.

Generally speaking, an increase in motor unit synchrony increases tremor amplitude and higher amplitude involves a more severe PD tremor (Elble et al. 1994; Elble and Randall 1976). Although difficult to assess, motor unit synchronization is likely one of the most accurate peripheral measures of pathological tremulous muscle activity (Holobar et al. 2012).

\section{Neuroimaging procedures}

\section{Neuroimage acquisition}


Participants were immobilized with a custom-fit blue bag vacuum mold (Medical Intelligence, Inc.) to prevent image artifacts. A strict criterion for head movement assessment was adopted (maximal absolute head movement less than $1.0 \mathrm{~mm}$ and $1.0^{\circ}$ in the $\mathrm{x}, \mathrm{y}$, and $\mathrm{z}$ directions). Neither patients nor healthy controls were excluded from the analysis due to this criterion. MRI data were acquired on each patient and control using a GE Signa 3.0 T scanner (General Electric Medical Systems, Milwaukee, WI) with a standard quadrature birdcage headcoil, using an axial 3D T1-weighted inversion-recovery fast gradient echo sequence $\left(\mathrm{TR}=5.0 \mathrm{~ms} ; \mathrm{TE}=2.2 \mathrm{~ms} ;\right.$ Flip Angle $\left.=12^{\circ} ; \mathrm{TI}=750 \mathrm{~ms} ; \mathrm{NEX}=1.0\right)$. A total of 176 contiguous 1 -mm slices were acquired with a $240 \times 240$ matrix with an in-plane resolution of $1 \times 1 \mathrm{~mm}$, resulting in isotropic voxels. Standard sequences of the MRI scans were checked before inclusion of a patient or control. Those with structural abnormalities in the brain, affecting gray or white matter, were excluded prior to the image analysis. MRI studies and imaging processing were performed by a neuroradiologist (JA-L, see acknowledgments) and a physicist (JAH-T see acknowledgments) who were blinded to the clinical diagnosis. In addition, those who interpreted the neurophysiological studies were blinded to MRI results (i.e., cortical thickness data).

\section{Neuroimage processing}

MRI images were processed to extract two types of information: volumetric features and cortical thickness features. Cortical reconstruction and volumetric segmentation was performed with the Freesurfer image analysis suite, which is documented and freely available for download online (http://surfer.nmr.mgh.harvard.edu/). Briefly, this processing includes motion correction and averaging (Reuter et al. 2010) of multiple volumetric T1 weighted 
images (when more than one is available), removal of non-brain tissue using a hybrid watershed/surface deformation procedure (Segonne et al. 2004), automated Talairach transformation, segmentation of the subcortical white matter and deep gray matter volumetric structures (including hippocampus, amygdala, caudate, putamen, ventricles) (Fischl et al. 2002; Fischl et al. 2004), intensity normalization (Sled et al. 1998), tessellation of the gray matter white matter boundary, automated topology correction (Segonne et al. 2007), and surface deformation following intensity gradients to optimally place the gray/white and gray/cerebrospinal fluid borders at the location where the greatest shift in intensity defines the transition to the other tissue class (Fischl and Dale 2000). Once the cortical models were complete, a number of deformable procedures could be performed for further data processing and analysis; these included surface inflation (Fischl et al. 1999), registration to a spherical atlas, which utilizes individual cortical folding patterns to match cortical geometry across subjects (Dale et al. 1999), fragmentation of the cerebral cortex into units based on gyral and sulcal structure (Desikan et al. 2006), and creation of a variety of surface based data including maps of curvature and sulcal depth. This method used both intensity and continuity information from the entire three dimensional MRI volume in segmentation and deformation procedures to produce representations of cortical thickness, calculated as the closest distance from the gray/white boundary to the gray/CSF (cerebrospinal fluid) boundary at each vertex on the tessellated surface (Fischl and Dale 2000). The maps were created using spatial intensity gradients across tissue classes and were therefore not simply reliant on absolute signal intensity. The cortical thickness features were average values for each region. Additionally, for each cortical region, the standard deviation of the cortical thickness was also calculated as a measure of roughness. We should keep in mind that the distribution 
of cortex thickness is not uniform by layer, neither is the variation in the thickness of the cortical layers proportional to the variation in the total thickness, nor is the location and progression of subtle cortical atrophy the same among individuals with the same neurodegenerative disease (Serrano et al. 2017). Hence, there is also a need for new more reliable variables to analyze the pattern of cortical thickness (Serrano et al. 2017). "Roughness" within a certain area may be therefore a promising metric to overcome these limitations (Serrano et al. 2017). An increase of roughness would imply a major cortical thinning (i.e., atrophy) (Serrano et al. 2017).

The above processing steps yielded 129 white matter and grey matter volumetric features of the whole brain (except for the cerebellum) and 152 cortical thickness features (average plus roughness, i.e. standard deviation of the thickness), according to the Desikan-Killiany atlas (Desikan et al. 2006), resulting in a total of 281 structural features from each subject.

\section{Statistical analyses}

Statistical analyses were performed in SPSS Version 23.0 (IBM Corp., NY, USA). IMUs and hdEMG metrics were linearly modelled by a subset of the 281 structural features extracted from MRI data together with demographic (age and sex) and clinical (UPDRS-m and time from PD onset) variables. For each metric from IMUs or hdEMG, the algorithm built any possible subset of the 281 structural features. For each subset, a Monte Carlo simulation algorithm was applied to find the optimum coefficients of the linear model (Lamnisos et al. 2013). Variables were added to the model under a criterion of prevention of overfitting, that is, assessing each new model update on a subset of the total sample. The mean square error 
of the linear model with respect to the actual values of the target variable was then calculated. Finally, the algorithm returned the model of the subset with the minimum error. For the present study, only the models of the target variables with an error lower than $20 \%$ were considered.

A correlation analysis was also carried out to find the pairwise relationships between IMUs and hdEMG, demographic (age and sex), clinical (UPDRS-m and time from PD onset), and structural (MRI) variables. The Pearson product-moment correlation coefficient was calculated for each pair under normality conditions (Shapiro-Wilk test). Spearman's rank correlation coefficient was calculated otherwise. A bootstrapping analysis (1000 samples) was applied to all the correlation calculations in order to prevent type-l error.(Henderson 2005) The correlations having a significance value of $p<0.05$ and a confidence interval with lower and upper bounds with the same sign as the correlation value were considered statistically significant.

\section{Results}

This study was nested within the NEUROTREMOR project (http://www.neuralrehabilitation.org/projects/neurotremor/), a project whose main aim was to validate technically, functionally and clinically, a novel system for understanding, providing diagnostic support for, and remotely managing tremors. Most of the PD patients who were eligible refused to participate because of lack of time - the study would have required that they come to the hospital several times for the performance of clinical, neurophysiological (magneto-electroencephalography and electromyography recordings), neuropsychological, 
and imaging evaluations. Given this constraint, of the 123 PD patients seen at outpatient neurology clinics of the University Hospital "12 de Octubre" in Madrid (Spain) from October 2012 to July 2013, only 34 were eligible for the study. Of these 34, 13 had complete neurophysiologic testing and an MRI procedure with cortical thickness data (see table 2). All of them were right-handed and had a dopamine nigrostriatal terminal defect, as assessed by [(123) I]FP-CIT single photon emission computed tomography. None of the participants were excluded due to neurological comorbidities or structural abnormalities on conventional MRI images, and none developed additional neurodegenerative diseases during the 5-year-followup period.

The final sample of 13 PD patients did not differ to a significant degree from the 17 healthy controls in terms of age, sex, and educational level (Table 2). The mean PD tremor duration was $5.5 \pm 2.9$ years and the UPDRS-m score was $12.4 \pm 5.4$ (Table 2).

\section{Linear regression models of hdEMG and IMUs activity from MRI data}

1) Mean logarithmic PSD of the tremor harmonics in AO, PO, WE, and RE tasks (IMU in the dominant tremor hand)

This linear model was determined by the following equation:

$y=-7.248 \times$ right caudal middle frontal thickness +

$14.455 \times$ left caudal middle frontal roughness -

\section{$2.631 \times$ left inferior parietal thickness}

Thus, the value of the mean logarithmic PSD of tremor harmonics in AO, PO, WE and RE tasks in the dominant tremor hand was chracterized by thickness-related variables from three 
cortical areas with a determination coefficient of 0.989 , as depicted in Figure 1. Please notice that some of the points in the graph overlap (also in subsequent analogue figures). Figure 1 also shows the relative contribution of the cortical variables to the model. The highest contribution was provided by the caudal middle frontal areas bilaterally (dorsal premotor cortices), although higher in the right hemisphere.

2) Mean logarithmic PSD of the tremor harmonics in AO, PO, WE, and RE tasks (IMU in the non-dominant tremor hand). This linear model was determined by the following equation:

$y=20.583 \times$ right cingulate lobe roughness -

\section{$2.106 \times$ right caudal middle frontal thickness -}

\subsection{2 left transverse temporal thickness}

The model comprised of three thickness-related variables with different contribution degrees as shown in Figure 2 and adjusted the actual values of the variable with a determination coefficient of 0.997 . In this case, cingulate and caudal middle frontal areas of the right hemisphere accounted for most of model variance.

3) Mean logarithmic PSD of the tremor harmonics in $A O, P O$, and WE tasks with respect to RE task (IMU in the dominant tremor hand)

The model obtained for this variable fitted with a coefficient of determination of 0.979 . It was determined by the following equation:

$y=-10.701 \times$ left occipital lobe thickness + $10.692 \times$ left posterior temporal roughness - 


\section{$4.952 \times$ left cingulate lobe roughness}

All the cortical areas of the model were located in the left hemisphere, with the mean contribution provided by the occipital lobe thickness, as shown in Figure 3.

4) Percentage of concurrent motor unit discharges in $A O$ with respect to $R E$ (EMG, in non-dominant tremor hand)

This metric directly measures the synchronization of motor units in $\mathrm{AO}$ task, normalized to synchronization of motor units in RE task. Therefore, it reflects the change in motor unit synchronization when patient moves from $A O$ to $R E$ task. This variable was linearly modeled by the following expresion:

$y=-0.001 \times$ left middle temporal volume +

\section{$2.551 \times$ left lateral orbitofrontal thickness}

In this case, the model was defined by two cortical variables in the left hemisphere (Figure 4). One of them refers to volume and the other to thickness. The determination coefficient of this model was 0.901 .

\section{MRI differences between PD patients and healthy controls}

Table 3 lists the brain anatomical areas that showed significant differences between ET patients and the healthy control group. Differences localized to certain cortical regions, which included cingulate cortex bilaterally, right superior parietal lobe, and both temporal lobes. Of note is that the bilateral cingulate cortices and the left temporal lobe were part of the linear models described above, which suggests an association between the areas 
associated with the diagnosis and the ones modulating (or modulated by) the severity of PD tremor.

\section{Correlations between IMUs and hdEMG metrics and MRI data, demographic and clinical features}

Table 4 shows the statistically significant correlations between IMUs or hdEMG metrics and MRI data, including demographic and clinical variables. All IMUs or hdEMG metrics with a significant correlation with any MRI data in Table 4 were successfully described by one of the linear models presented above. In addition, all MRI data in Table 4 were part of the linear models of their correlated IMUs and hdEMG metrics, except for the mean logarithmic PSD of the tremor harmonics in $\mathrm{AO}, \mathrm{PO}, \mathrm{WE}$, and RE tasks (IMU in the dominant tremor hand) (first colum). In this latter case, the IMU metric significantly correlated with the roughness of the left cingulate cortex, which was not present in the corresponding linear model. Only one clinical variable was correlated with a MRI structure: the time from tremor onset was inversely correlated with the volume of the left middle temporal cortex. This structural variable was also present in the model 4 (percentage of motor unit discharges in $A O$ with respect to $R E$ in the non-dominant tremor hand) (Figure 4).

\section{Discussion}

To our knowledge, the present study provides the first evidence that changes in frontoparietal circuit areas that regulate movement sequencing is strongly associated with increased severity of tremor in PD patients. Increased motor unit synchronization (i.e., more 
severe tremor) was associated with cortical changes (i.e., atrophy) in widespread cortical areas, including caudal middle frontal regions bilaterally (dorsal premotor cortices), left inferior parietal lobe (posterior parietal cortex), left lateral orbitofrontal cortex, cingulate cortex bilaterally, left posterior and transverse temporal cortex, and left occipital lobe, as well as reduced left middle temporal volume. The majority of these areas are involved in controlling movement sequencing (Bengtsson et al. 2004; Rushworth et al. 2001b).

A sizable number of studies highlight the key role of a neural circuit involving frontal and parietal areas in movement sequencing (Bengtsson et al. 2004; Bortoletto and Cunnington 2010; Rushworth et al. 2001b). For example, in a PET study, enhanced activity in the cingulate motor area, dorsal lateral premotor area and intraparietal sulcus was observed when participants specifically attended to sequencing movements (Rushworth et al. 2001b). Further, patients with left parietal damage had problems performing sequences of movements (Rushworth et al. 1997). In line with this, sequence preparation was altered by transcranial magnetic stimulation of the left parietal cortex (Rushworth et al. 2001a).

The cortical changes observed in the caudal middle frontal gyrus (dorsal premotor cortex) bilaterally and posterior parietal cortex may be associated with tremor genesis given that these areas act on motor output through direct or indirect connections with the primary motor cortex and the spinal cord (Karabanov et al. 2012; Picard and Strick 2001). Specifically, it is well established that the dorsal premotor cortex plays a central role in planning and selecting movements (Deiber et al. 1991), and is thought to be a critical node in the motor learning network (Hardwick et al. 2013). The posterior parietal cortex, including the inferior parietal lobule, has been shown to be involved in the preparation and redirection of movements (Rushworth et al. 2003), as well as in intention to perform specific motor acts (Desmurget et 
al. 2009). Besides, the parietal cortex has strong interactions with extrastriate body area (Zimmermann et al. 2017). This area, which is located in the lateral occipital cortex at the posterior end of the inferior temporal sulcus, is well-known to respond to visual processing of static and moving human bodies even in the absence of visual feedback from the limb (Astafiev et al. 2004; Lingnau and Downing 2015). In addition, the extrastriate body area responds not only during the perception of other people's body parts, but also during goaldirected movements of the observer's body parts (Astafiev et al. 2004; Lingnau and Downing 2015). On the other hand, it is known that the lateral occipitotemporal cortex plays important roles in the perception, understanding and production of action (Lingnau and Downing 2015). Consequently, a deterioration of the above-mentioned areas could plausibly be related to motor disorders (i.e., tremor).

Of additional interest, we found that the left lateral orbitofrontal cortex was affected. This structure is thought to be involved in goal-directed behavior and is affected in PD patients (Marinelli et al. 2015). In a recent study of untreated patients in the early stages of PD, the reaction time during a choice reaction time paradigm was inversely correlated to dopaminergic activity as measured by $18 \mathrm{~F}-\mathrm{DOPA}$ PET in the left lateral orbitofrontal cortex (Marinelli et al. 2015). This finding underscores the role of dopamine in the lateral orbitofrontal cortex in the early stages of PD, supporting a relation between the compensatory cortical dopamine and movement preparation (Marinelli et al. 2015). Interestingly, cingulate cortex changes bilaterally was associated with increased PD tremor severity. The cingulate cortex may also be involved in some way with the modulation of movement sequencing since the anterior cingulate cortex plays a key role in relating actions 
to their consequences, both positive reinforcement outcomes and errors, and in guiding decisions about which actions are worth making (Rushworth et al. 2004).

Taken together, the cortical changes observed in these areas may indicate that PD patients might be more inefficient in integrating multi-modal information in this fronto-parietal network to produce an output that reflects the selection, preparation, and execution of movements (Wise et al. 1997), which would result in tremor generation.

Cortical changes could be explained by sustained neuronal activation and subsequent neuronal damage or loss, but these could alternatively be interpreted in terms of changes in energy demand in both sensory and motor neural loops (Mangia et al. 2007). Increased motor unit synchronization is clear evidence of muscle fatigue (central and/or peripheral) (Boyas and Guevel 2011), and hence, higher energy demand, which would ultimate facilitate neuronal damage with the subsequent cortical thinning (i.e., atrophy). This hypothesis must be investigated further.

It is worth mentioning that different variables of different nature (IMUs, hdEMG or clinical features) were correlated with different anatomical regions and features of the cortical anatomy. This is likely because they are measuring different phenomena, despite all being related to tremor severity. IMUs are measuring the amplitude of tremor, i.e. how much muscles are contracting during tremor. Motor unit firing extracted from hdEMG is measuring the resources used to perform the tremorous muscle contractions as well as the stiffness of muscles during contractions (the more stiffness the more motor units concurrent firing). Time from onset is a clinical feature directly, but not linearly, associated with tremor severity, according to visually scored tremor scales, and thus subjective to the clinician. Given the 
former statements, it is rather expected that the variables of different nature were associated with different brain structures.

The study was not without limitations. First, the sample size was relatively small. However, we observed robust correlations. Notwithstanding, it would be important to replicate these findings in a larger sample, as small samples may be subject to spurious findings. Second, the diagnosis of PD was based on clinical criteria and further supported by altered [(123) I]FP-CIT SPECT results. However, none of the PD patients had post-mortem assessments. Finally, the extremely high values of the coefficients of determination $\left(R^{2} \geq\right.$ 0.979) in the linear models obtained might be caused by the presence of one or two outlier values. However, the absence of such outliers would still keep high values of $R^{2}$, as can be deduced from graphs in Figures 1,2 , and $3\left(R^{2}=0.953, R^{2}=0.992\right.$, and $R^{2}=0.927$, respectively without outliers) .

In closing, given that the accurate control of movement sequencing is important for motor behavior programs and that most these thinned cortical areas mainly overlap with those involved in controlling motor sequencing, our results support the Albert's classical hypothesis (Alberts 1972) that PD tremor may be the result of an involuntary activation of a program of motor behavior ordinarily used in the genesis of rapid voluntary alternating movements. Notwithsanding, our findings should be considered only as a preliminary result, more for exploratory purposes and hypothesis generation than for a solid conclusion. Additional studies with larger samples are required. 


\section{Competing interests}

The authors declare no competing financial interests.

\section{Authors Roles:}

Dr. Benito-León (jbenitol67@gmail.com) collaborated in: 1) the conception, organization of the research project; 2) the statistical analysis design, and 3) the writing of the manuscript first draft and the review and critique of the manuscript.

Dr. Serrano (iignacio.serrano@csic.es) collaborated in: 1) the conception, organization and execution of the research project; 2) the statistical analysis design, and; 3) and the review and critique of the manuscript.

Dr. Louis (elan.louis@yale.edu) collaborated in: 1) the conception, organization of the research project; and 2) the review and critique of the manuscript.

Dr. Holobar (ales.holobar@uni-mb.si) collaborated in: 1) the conception, organization of the research project; and 2) the review and critique of the manuscript.

Dr. Romero (juanpa5@hotmail.com) collaborated in: 1) the conception, organization of the research project; and 2) the review and critique of the manuscript.

Dr. Povalej-Bržan (petrapovalej@gmail.com) collaborated in: 1) the conception, organization of the research project; and 2) the review and critique of the manuscript.

Dr. Bermejo-Pareja (fbermejop2013@yahoo.es) collaborated in: 1) the conception, 
organization of the research project; and 2) the review and critique of the manuscript.

Dr. del Castillo (md.delcastillo@csic.es) collaborated in: 1) the conception, organization of the research project; 2) the statistical analysis design; and 3) the review and critique of the manuscript.

Dr.Posada (ignaciojavier.posada@salud.madrid.org) collaborated in: 1) the conception, organization of the research project; and 2) the review and critique of the manuscript.

Dr. Rocon (e.rocon@csic.es) collaborated in: 1) the conception, organization of the research project; and 2) the review and critique of the manuscript.

\section{Disclosures:}

Dr. Benito-León reports no disclosures.

Dr. Serrano reports no disclosures.

Dr. Louis reports no disclosures.

Dr. Holobar reports no disclosures.

Dr. Romero reports no disclosures.

Dr. Povalej-Bržan reports no disclosures.

Dr. Bermejo-Pareja reports no disclosures. 
Dr. del Castillo reports no disclosures.

Dr. Posada reports no disclosures.

Dr. Rocon reports no disclosures.

\section{Acknowledgments and Funding}

This research was supported by FEDER funds. Dr. Benito-León is supported by the National Institutes of Health, Bethesda, MD, USA (NINDS \#R01 NS39422), the Commission of the European Union (grant ICT-2011-287739, NeuroTREMOR), the Ministry of Economy and Competitiveness (grant RTC-2015-3967-1, NetMD—platform for the tracking of movement disorder), and the Spanish Health Research Agency (grant FIS PI12/01602 and grant FIS PI16/00451). Drs. Serrano, Romero, del Castillo and Rocon are supported by the Spanish Ministry of Economy and Competitiveness (grant DPI-2015-68664-C4-1-R, NeuroMOD). Dr. Louis has received research support from the National Institutes of Health: NINDS \#R01 NS094607 (principal investigator), NINDS \#R01 NS085136 (principal investigator), NINDS \#R01 NS073872 (principal investigator), NINDS \#R01 NS085136 (principal investigator) and NINDS \#R01 NS088257 (principal investigator). He has also received support from the Claire O'Neil Essential Tremor Research Fund (Yale University). Dr. Holobar is supported by the Slovenian Research Agency (project J2-7357 - Exact quantification of muscle control strategies and co-activation patterns in robot-assisted rehabilitation of hemiparetic patients and Programme funding P2-0041). Drs. Holobar, Romero, Povalej-Bržan, Bermejo-Pareja, Posada and Rocon are supported by the Commission of the European Union (grant ICT- 


\section{1-287739, NeuroTREMOR). We thank Drs. Juan Álvarez-Linera and José Antonio \\ Hernández-Tamames for their assistance to the project.}

\section{References}

Alberts WW. 1972. A simple view of Parkinsonian tremor. Electrical stimulation of cortex adjacent to the Rolandic fissure in awake man. Brain Res 44(2):357-369.

American Psychiatric A. 1994. Diagnostic and Statistical Manual of Mental Disorders DSM-IV. Washington.

Antonini A, Moeller JR, Nakamura T, Spetsieris P, Dhawan V, Eidelberg D. 1998. The metabolic anatomy of tremor in Parkinson's disease. Neurology 51(3):803-810.

Astafiev SV, Stanley CM, Shulman GL, Corbetta M. 2004. Extrastriate body area in human occipital cortex responds to the performance of motor actions. Nat Neurosci 7(5):542-548.

Bengtsson SL, Ehrsson HH, Forssberg H, Ullen F. 2004. Dissociating brain regions controlling the temporal and ordinal structure of learned movement sequences. Eur J Neurosci 19(9):2591-2602.

Benito-Leon J, Louis ED, Romero JP, Hernandez-Tamames JA, Manzanedo E, Alvarez-Linera J, Bermejo-Pareja F, Posada I, Rocon E. 2015. Altered Functional Connectivity in Essential Tremor: A Resting-State fMRI Study. Medicine (Baltimore) 94(49):e1936.

Bortoletto M, Cunnington R. 2010. Motor timing and motor sequencing contribute differently to the preparation for voluntary movement. Neuroimage 49(4):3338-3348.

Boyas S, Guevel A. 2011. Neuromuscular fatigue in healthy muscle: underlying factors and adaptation mechanisms. Ann Phys Rehabil Med 54(2):88-108.

Br P, \#x17e, an P, Gallego JA, Romero JP, Glaser V, Rocon E, Benito-Le, \#xf3, n J, Bermejo-Pareja F, Posada IJ, Holobar A. 2017. New Perspectives for Computer-Aided Discrimination of Parkinson\&\#x2019; Disease and Essential Tremor. Complexity 2017:17.

Chen W, Hopfner F, Becktepe JS, Deuschl G. 2017. Rest tremor revisited: Parkinson's disease and other disorders. Transl Neurodegener 6:16.

Dale AM, Fischl B, Sereno MI. 1999. Cortical surface-based analysis. I. Segmentation and surface reconstruction. Neuroimage 9(2):179-194.

De Luca CJ, Roy AM, Erim Z. 1993. Synchronization of motor-unit firings in several human muscles. J Neurophysiol 70(5):2010-2023.

Deiber MP, Passingham RE, Colebatch JG, Friston KJ, Nixon PD, Frackowiak RS. 1991. Cortical areas and the selection of movement: a study with positron emission tomography. Exp Brain Res 84(2):393-402.

Deiber MP, Pollak P, Passingham R, Landais P, Gervason C, Cinotti L, Friston K, Frackowiak R, Mauguiere F, Benabid AL. 1993. Thalamic stimulation and suppression of parkinsonian tremor. Evidence of a cerebellar deactivation using positron emission tomography. Brain 116 ( Pt 1):267-279.

Deng X, Tang CY, Zhang J, Zhu L, Xie ZC, Gong HH, Xiao XZ, Xu RS. 2016. The cortical thickness correlates of clinical manifestations in the mid-stage sporadic Parkinson's disease. Neurosci Lett 633:279-289.

Desikan RS, Segonne F, Fischl B, Quinn BT, Dickerson BC, Blacker D, Buckner RL, Dale AM, Maguire RP, Hyman BT, Albert MS, Killiany RJ. 2006. An automated labeling system for subdividing the human cerebral cortex on MRI scans into gyral based regions of interest. Neuroimage 31(3):968-980.

Desmurget M, Reilly KT, Richard N, Szathmari A, Mottolese C, Sirigu A. 2009. Movement intention after parietal cortex stimulation in humans. Science 324(5928):811-813.

Deuschl G, Bain P, Brin M. 1998. Consensus statement of the Movement Disorder Society on Tremor. Ad Hoc Scientific Committee. Mov Disord 13 Suppl 3:2-23.

Duval C, Daneault JF, Hutchison WD, Sadikot AF. 2016. A brain network model explaining tremor in Parkinson's disease. Neurobiol Dis 85:49-59.

Elble RJ, Higgins C, Leffler K, Hughes L. 1994. Factors influencing the amplitude and frequency of essential tremor. Mov Disord 9(6):589-596. 
Elble RJ, Randall JE. 1976. Motor-unit activity responsible for 8- to 12-Hz component of human physiological finger tremor. J Neurophysiol 39(2):370-383.

Fischl B, Dale AM. 2000. Measuring the thickness of the human cerebral cortex from magnetic resonance images. Proc Natl Acad Sci U S A 97(20):11050-11055.

Fischl B, Salat DH, Busa E, Albert M, Dieterich M, Haselgrove C, van der Kouwe A, Killiany R, Kennedy D, Klaveness S, Montillo A, Makris N, Rosen B, Dale AM. 2002. Whole brain segmentation: automated labeling of neuroanatomical structures in the human brain. Neuron 33(3):341-355.

Fischl B, Salat DH, van der Kouwe AJ, Makris N, Segonne F, Quinn BT, Dale AM. 2004. Sequence-independent segmentation of magnetic resonance images. Neuroimage 23 Suppl 1:S69-84.

Fischl B, Sereno MI, Dale AM. 1999. Cortical surface-based analysis. II: Inflation, flattening, and a surface-based coordinate system. Neuroimage 9(2):195-207.

Glaser V, Farina D, Holobar A. 2017. Simulations of high-density surface electromyograms in dynamic muscle contractions. Conf Proc IEEE Eng Med Biol Soc 2017:3453-3456.

Hardwick RM, Rottschy C, Miall RC, Eickhoff SB. 2013. A quantitative meta-analysis and review of motor learning in the human brain. Neuroimage 67:283-297.

Henderson AR. 2005. The bootstrap: a technique for data-driven statistics. Using computer-intensive analyses to explore experimental data. Clin Chim Acta 359(1-2):1-26.

Holobar A, Farina D, Gazzoni M, Merletti R, Zazula D. 2009. Estimating motor unit discharge patterns from high-density surface electromyogram. Clin Neurophysiol 120(3):551-562.

Holobar A, Glaser V, Gallego JA, Dideriksen JL, Farina D. 2012. Non-invasive characterization of motor unit behaviour in pathological tremor. J Neural Eng 9(5):056011.

Holobar A, Minetto MA, Farina D. 2014. Accurate identification of motor unit discharge patterns from high-density surface EMG and validation with a novel signal-based performance metric. J Neural Eng 11(1):016008.

Hughes AJ, Daniel SE, Kilford L, Lees AJ. 1992. Accuracy of clinical diagnosis of idiopathic Parkinson's disease: a clinico-pathological study of 100 cases. J Neurol Neurosurg Psychiatry 55(3):181-184.

Karabanov A, Jin SH, Joutsen A, Poston B, Aizen J, Ellenstein A, Hallett M. 2012. Timing-dependent modulation of the posterior parietal cortex-primary motor cortex pathway by sensorimotor training. J Neurophysiol 107(11):31903199.

Lamnisos D, Griffin JE, Steel MFJ. 2013. Adaptive Monte Carlo for Bayesian Variable Selection in Regression Models. Journal of Computational and Graphical Statistics 22(3):729-748.

Lewis MM, Galley S, Johnson S, Stevenson J, Huang X, McKeown MJ. 2013. The role of the cerebellum in the pathophysiology of Parkinson's disease. Can J Neurol Sci 40(3):299-306.

Lingnau A, Downing PE. 2015. The lateral occipitotemporal cortex in action. Trends Cogn Sci 19(5):268-277.

Louis ED, Levy G, Cote LJ, Mejia H, Fahn S, Marder K. 2001. Clinical correlates of action tremor in Parkinson disease. Arch Neurol 58(10):1630-1634.

Lozza C, Marie RM, Baron JC. 2002. The metabolic substrates of bradykinesia and tremor in uncomplicated Parkinson's disease. Neuroimage 17(2):688-699.

Mangia S, Tkac I, Gruetter R, Van de Moortele PF, Maraviglia B, Ugurbil K. 2007. Sustained neuronal activation raises oxidative metabolism to a new steady-state level: evidence from 1H NMR spectroscopy in the human visual cortex. J Cereb Blood Flow Metab 27(5):1055-1063.

Marinelli L, Piccardo A, Mori L, Morbelli S, Girtler N, Castaldi A, Picco A, Trompetto C, Ghilardi MF, Abbruzzese G, Nobili F. 2015. Orbitofrontal (18) F-DOPA Uptake and Movement Preparation in Parkinson's Disease. Parkinsons Dis 2015:180940.

Martinez-Martin P, Gil-Nagel A, Gracia LM, Gomez JB, Martinez-Sarries J, Bermejo F. 1994. Unified Parkinson's Disease Rating Scale characteristics and structure. The Cooperative Multicentric Group. Mov Disord 9(1):76-83.

Mure H, Hirano S, Tang CC, Isaias IU, Antonini A, Ma Y, Dhawan V, Eidelberg D. 2011. Parkinson's disease tremorrelated metabolic network: characterization, progression, and treatment effects. Neuroimage 54(2):1244-1253.

Parker F, Tzourio N, Blond S, Petit H, Mazoyer B. 1992. Evidence for a common network of brain structures involved in parkinsonian tremor and voluntary repetitive movement. Brain Res 584(1-2):11-17.

Picard N, Strick PL. 2001. Imaging the premotor areas. Curr Opin Neurobiol 11(6):663-672.

Pirker W. 2003. Correlation of dopamine transporter imaging with parkinsonian motor handicap: how close is it? Mov Disord 18 Suppl 7:S43-51. 
Qasim SE, de Hemptinne C, Swann NC, Miocinovic S, Ostrem JL, Starr PA. 2016. Electrocorticography reveals beta desynchronization in the basal ganglia-cortical loop during rest tremor in Parkinson's disease. Neurobiol Dis 86:177-186.

Rajput AH, Voll A, Rajput ML, Robinson CA, Rajput A. 2009. Course in Parkinson disease subtypes: A 39-year clinicopathologic study. Neurology 73(3):206-212.

Reuter M, Rosas HD, Fischl B. 2010. Highly accurate inverse consistent registration: a robust approach. Neuroimage 53(4):1181-1196.

Rushworth MF, Ellison A, Walsh V. 2001a. Complementary localization and lateralization of orienting and motor attention. Nat Neurosci 4(6):656-661.

Rushworth MF, Johansen-Berg H, Gobel SM, Devlin JT. 2003. The left parietal and premotor cortices: motor attention and selection. Neuroimage 20 Suppl 1:S89-100.

Rushworth MF, Krams M, Passingham RE. 2001b. The attentional role of the left parietal cortex: the distinct lateralization and localization of motor attention in the human brain. J Cogn Neurosci 13(5):698-710.

Rushworth MF, Nixon PD, Renowden S, Wade DT, Passingham RE. 1997. The left parietal cortex and motor attention. Neuropsychologia 35(9):1261-1273.

Rushworth MF, Walton ME, Kennerley SW, Bannerman DM. 2004. Action sets and decisions in the medial frontal cortex. Trends Cogn Sci 8(9):410-417.

Segonne F, Dale AM, Busa E, Glessner M, Salat D, Hahn HK, Fischl B. 2004. A hybrid approach to the skull stripping problem in MRI. Neuroimage 22(3):1060-1075.

Segonne F, Pacheco J, Fischl B. 2007. Geometrically accurate topology-correction of cortical surfaces using nonseparating loops. IEEE Trans Med Imaging 26(4):518-529.

Serrano JI, Romero JP, Castillo MDD, Rocon E, Louis ED, Benito-Leon J. 2017. A data mining approach using cortical thickness for diagnosis and characterization of essential tremor. Sci Rep 7(1):2190.

Sled JG, Zijdenbos AP, Evans AC. 1998. A nonparametric method for automatic correction of intensity nonuniformity in MRI data. IEEE Trans Med Imaging 17(1):87-97.

Timmermann L, Gross J, Dirks M, Volkmann J, Freund HJ, Schnitzler A. 2003. The cerebral oscillatory network of parkinsonian resting tremor. Brain 126(Pt 1):199-212.

Volkmann J, Joliot M, Mogilner A, Ioannides AA, Lado F, Fazzini E, Ribary U, Llinas R. 1996. Central motor loop oscillations in parkinsonian resting tremor revealed by magnetoencephalography. Neurology 46(5):1359-1370.

Willingham DB. 1998. A neuropsychological theory of motor skill learning. Psychol Rev 105(3):558-584.

Wise SP, Boussaoud D, Johnson PB, Caminiti R. 1997. Premotor and parietal cortex: corticocortical connectivity and combinatorial computations. Annu Rev Neurosci 20:25-42.

Zach H, Dirkx M, Bloem BR, Helmich RC. 2015. The Clinical Evaluation of Parkinson's Tremor. J Parkinsons Dis 5(3):471-474.

Zimmermann M, Mars RB, de Lange FP, Toni I, Verhagen L. 2017. Is the extrastriate body area part of the dorsal visuomotor stream? Brain structure \& function. 
Table 1: Tremor-triggering tasks performed during recordings of wrist movement and hdEMG data. Each task was performed 3 times.

\begin{tabular}{|c|c|}
\hline Task & Description \\
\hline Rest (RE) & $\begin{array}{l}\text { The patient rested both arms, which were completely relaxed anc } \\
\text { supported either on the armrests or on the patient's lap, depending } \\
\text { on what he/she reported to be most comfortable. }\end{array}$ \\
\hline Arms outstretched (AO) & $\begin{array}{l}\text { The patient kept his/her arms outstretched, parallel to the ground, } \\
\text { with the palms down and the fingers apart. }\end{array}$ \\
\hline $\begin{array}{l}\text { Arms outstretched with } \\
\text { weights (WE) }\end{array}$ & $\begin{array}{l}\text { The same as the } \mathrm{AO} \text { task but with one-kilogram weight fixed to both } \\
\text { hands. }\end{array}$ \\
\hline $\begin{array}{l}\text { Arms supported + postural } \\
\text { tremor elicited (PO) }\end{array}$ & $\begin{array}{l}\text { The same as RE task, but with hands and fingers extended agains } \\
\text { gravity. }\end{array}$ \\
\hline
\end{tabular}


Table 2: Demographic and clinical characteristics of Parkinson's disease patients and healthy control group.

\begin{tabular}{|c|c|c|c|c|}
\hline & & $\begin{array}{l}\text { Healthy controls }(\mathrm{N}= \\
17)\end{array}$ & $\begin{array}{c}\text { Parkinson's disease } \\
\text { patients }(\mathrm{N}=13)\end{array}$ & $p$ value \\
\hline \multicolumn{2}{|l|}{ Sex (women) } & $10(58.8 \%)$ & $9(69.2 \%)$ & $x(1)=0.475, p=0.491$ \\
\hline \multicolumn{2}{|l|}{ Age in years } & $64.1 \pm 11.9$ (39 to 81$)$ & $65.6 \pm 10.1$ (45 to 78$)$ & $t(28)=-1.008, p=0.322$ \\
\hline \multirow{4}{*}{$\begin{array}{l}\text { Educational } \\
\text { level }\end{array}$} & $\begin{array}{l}\text { Reading and } \\
\text { Writing }\end{array}$ & $3(17.6 \%)$ & $4(30.8 \%)$ & \multirow{4}{*}{$x(3)=0.2 .431, p=0.488$} \\
\hline & Primary & $4(23.5 \%)$ & $2(15.4 \%)$ & \\
\hline & Secondary & $6(35.3 \%)$ & $2(15.4 \%)$ & \\
\hline & University & $4(23.5 \%)$ & $5(38.5 \%)$ & \\
\hline \multicolumn{2}{|l|}{ UPDRS-m ${ }^{1}$} & - & $12.4 \pm 5.4$ (7 to 25$)$ & \\
\hline \multicolumn{2}{|c|}{$\begin{array}{l}\text { Time from Parkinson's } \\
\text { disease onset in years }\end{array}$} & - & $5.5 \pm 2.9(2$ to 13$)$ & \\
\hline
\end{tabular}

Age and clinical values are expressed as mean \pm standard deviation (range). The rest of values are expressed as number of participants (percentage of condition). Student's $t$ test was used for comparison of continuous data and the chi-square test for sex and level of education.

${ }^{1}$ Motor section of the Unified Parkinson's Disease Rating Scale. 
Table 3: Statistically significant differences (t-test, $p<0.05)$ of MRI morphometry features between the PD patients and the healthy control group.

\begin{tabular}{|l|c|c|}
\hline & $\begin{array}{c}\text { Control } \\
\text { participants } \\
\mathbf{( N = 1 7 )}\end{array}$ & $\begin{array}{c}\text { PD Patients } \\
\text { (N = 13) }\end{array}$ \\
\hline Intracraneal volume & $1599478.67 \pm 186692.06$ & $1765667.38 \pm 192375.64$ \\
\hline Cerebrospinal fluid & $541393.37 \pm 164518.23$ & $657928.45 \pm 121967.77$ \\
\hline Left middle temporal volume & $9384 \pm 1097$ & $8156 \pm 616$ \\
\hline Left temporal pole volume & $2570 \pm 431$ & $2892 \pm 366$ \\
\hline Left posterior cingulate volume & $2751 \pm 408$ & $3146 \pm 461$ \\
\hline Left posterior temporal thickness & $2.6189 \pm 0.1253$ & $2.4763 \pm 0.1723$ \\
\hline Left posterior temporal roughness & $0.4593 \pm .0613$ & $0.5375 \pm 0.0748$ \\
\hline Right superior parietal volume & $17585 \pm 3982$ & $20035 \pm 1813$ \\
\hline Right temporal pole volume & $2514 \pm 359$ & $2810 \pm 378$ \\
\hline Right rostral anterior cingulate volume & $1973 \pm 245$ & $2269 \pm 490$ \\
\hline Right cingulate lobe roughness & $0.6939 \pm .0638$ & $0.7521 \pm 0.0689$ \\
\hline Right caudal anterior cingulate roughness & $0.6581 \pm .0946$ & $0.7453 \pm 0.0860$ \\
\hline
\end{tabular}

Values are expressed as mean \pm standard deviation. 
Table 4: Matrix of correlations among IMUs and hdEMG metrics and MRI data, and clinical features.

\begin{tabular}{|c|c|c|c|c|c|}
\hline & & $\begin{array}{l}\text { Mean logarithmic PSD of } \\
\text { the tremor harmonics in } \\
\text { AO, PO, WE, and RE tasks } \\
\text { (IMU in the dominant } \\
\text { tremor hand) }\end{array}$ & $\begin{array}{c}\text { Mean logarithmic PSD of } \\
\text { the tremor harmonics in } \\
\mathrm{AO}, \mathrm{PO} \text { and WE tasks with } \\
\text { respect to mean } \\
\text { logarithmic PSD of tremor } \\
\text { harmonics in RE task (IMU } \\
\text { in the dominant tremor } \\
\text { hand) }\end{array}$ & $\begin{array}{l}\text { Percentage of concurrent } \\
\text { motor unit discharges in } \\
A O / \text { Percentage of } \\
\text { concurrent motor unit } \\
\text { discharges in RE (hdEMG } \\
\text { in the non-dominant tremor } \\
\text { hand) }\end{array}$ & $\begin{array}{c}\text { Time from } \\
\text { Parkinson's } \\
\text { disease onset }\end{array}$ \\
\hline \multirow{2}{*}{$\begin{array}{l}\text { Left occipital lobe } \\
\text { thickness }\end{array}$} & Coefficient & & $-0.767^{*}$ & & \\
\hline & $p$ value & & 0.044 & & \\
\hline \multirow{2}{*}{$\begin{array}{l}\text { Left cingulate cortex } \\
\text { roughness }\end{array}$} & Coefficient & $0.857^{\star *}$ & $0.793^{*}$ & & \\
\hline & $p$ value & 0.014 & 0.033 & & \\
\hline \multirow{2}{*}{$\begin{array}{l}\text { Right cingulate cortex } \\
\text { roughness }\end{array}$} & Coefficient & & $0.786^{\star *}$ & & \\
\hline & $p$ value & & 0.036 & & \\
\hline \multirow{2}{*}{$\begin{array}{l}\text { Left middle temporal } \\
\text { volume }\end{array}$} & Coefficient & & & & $-0.777^{\star \star}$ \\
\hline & $p$ value & & & & 0.040 \\
\hline \multirow{2}{*}{$\begin{array}{l}\text { Left lateral orbitofrontal } \\
\text { thickness }\end{array}$} & Coefficient & & & $0.837^{*}$ & \\
\hline & $p$ value & & & 0.019 & \\
\hline
\end{tabular}

${ }^{*}$ Pearson product-moment correlation coefficient; ** Spearman's rank correlation coefficient.

Arms outstretched (AO); arms supported + postural tremor elicited (PO); arms outstretched with weights (WE); rest (RE); high-

density surface electromyography (hdEMG); inertial measurement unit (IMU); Welch's power spectral density (PSD). 
Figure 1: Left: Linearly modeled versus actual values of the mean logarithmic PSD of the tremor harmonics in $A O, P O$, WE and RE tasks in the dominant tremor hand. Right: Cortical areas comprising the descriptive model and their relative contribution. L: Left hemisphere; R: Right hemisphere.

Figure 2: Left: Linearly modeled versus actual values of the mean logarithmic PSD of the tremor harmonics in $A O, P O$, WE and RE tasks in the non-dominant tremor hand. Right: Cortical areas comprising the descriptive model and their relative contribution. L: Left hemisphere; R: Right hemisphere.

Figure 3: Left: Linearly modeled versus actual values of the mean logarithmic PSD of the tremor harmonics in $A O, P O$, WE tasks with respect to RE task in the dominant tremor hand. Right: Cortical areas comprising the descriptive model and their relative contribution. L: Left hemisphere; R: Right hemisphere.

Figure 4: Left: Linearly modeled versus actual values of the percentage of concurrent motor unit discharges in $A O$ task with respect to RE task in the non-dominant tremor hand. Right: Cortical areas comprising the descriptive model and their relative contribution. L: Left hemisphere; R: Right hemisphere. 